\title{
Travel Time Reliability of Bus Operation in Heterogeneous Traffic Conditions of Dar es Salaam City, Tanzania
}

\author{
Prosper S. Nyaki ${ }^{1 *}$, Hannibal Bwire ${ }^{2}$ and Nurdin K. Mushule ${ }^{2}$
}

${ }^{1}$ National Institute of Transport, Department of Logistics and Transport Studies, P.O. Box 705, Dar es Salaam Tanzania; E-mail: nyakiprosper@yahoo.com

${ }^{2}$ University of Dar es Salaam, College of Engineering and Technology, Department of Transportation and Geotechnical Engineering, P.O. Box 35131, Dar es Salaam; E-mail: bwirehj@gmail.com,mushule@udsm.ac.tz

\section{*Corresponding Author: Prosper Sebastian Nyaki}

Received: 29 September 2020; Revised: 14 October 2020; Accepted: 19 October 2020; Published: 30 November 2020

\begin{abstract}
The assessment of travel time reliability enables precise prediction of travel times, better activity scheduling and decisions for all users of the road network. Furthermore, it helps to monitor traffic flow as a crucial strategy for reducing traffic congestion and ensuring high-quality service in urban roads. Travel time reliability is a useful reference tool for evaluating transport service quality, operating costs and system efficiency. However, many analyses of travel time reliability do not provide true travel variation under heterogeneous traffic flow conditions where traffic flow is a mixture of motorized and non-motorized transport. This study analysed travel time reliability under heterogeneous traffic conditions. The travel reliabilities focused on passenger waiting time at bus stops, in-vehicle travel time, and delay time at intersections which were analysed using buffer time, standard deviation, coefficient of variation, and planning time. The data used were obtained from five main bus routes in Dar es Salaam. The results indicate low service reliability in the outbound directions compared to inbound directions. They also intend to raise awareness of policy-makers about the situation and to make them shift from expanding road networks towards optimising road operations.
\end{abstract}

Keywords: Travel time reliability, heterogeneous traffic flow conditions, travel buffer time, planning time, travel time evaluation

\section{Introduction}

Travel time variability is a key indicator that road users take into account when making basic travel decisions such as choosing the mode of transport, transport route, and departure time [1]. 
Furthermore, travel time reliability is valuable for engineers as well as for planning, evaluating and managing transport systems. It is also an essential factor for assessing traffic flow that leads to increasing road capacity and formulating appropriate transport policy [2,3]. The factors that affect travel time reliability are unpredictable due to uncertainties of the demand for transport services. Most researchers have revealed that travel time reliability is affected by several factors such as weather conditions, natural disasters, congestion, road maintenance, traffic control, road accidents, in-vehicle travel time, waiting time at the intersections and bus stops [4-6]. However, this study focuses on in-vehicle travel time, waiting time at the intersections and bus stops data to determine travel time reliability in Dar es Salaam city.

Travel time variability is used as an important factor for evaluating congestion in the urban transportation system in developed countries [7-9]. Literary research shows that a few or no studies have been conducted in developing countries including Tanzania on analysing urban travel time reliability $[7,9,10]$. In Tanzania, analysing travel time reliability is more complicated because of heterogeneous traffic flow conditions which comprise both motorized and non-motorized vehicles that share the same road space. Therefore, this study analyses the travel time reliability-based urban bus operational characteristics of developing countries in heterogeneous traffic conditions. The reliability of in-vehicle travel time, waiting time at intersections and bus stops were evaluated by applying standard deviation, coefficient of variation, buffer time, and planning time. Travellers and transport planners have been witnessing high travel time variations at the bus stops and intersections resulting in long waiting time and in-vehicle time. Clear understanding of the travel time variability will help travellers, transport operators and transport planners to make rational decisions on how service quality and road bottlenecks can be improved.

The analysis of travel time reliability enables policy-makers, transport operators and travellers to understand road bottlenecks and other significant attributes of urban congestions [11]. Travel time reliability estimated on the basis of traffic flow, road, capacity, weather conditions and accident rate indicate that traffic flow and road accidents are closely related to travel time variation [12]. Furthermore, estimated travel time reliability using Buffer time and Delft skewers from the fitted Burr parameters, can be used to measure the performance of urban transportation facilities [13]. The literature [14] analysed urban travel time reliability in bus lanes using three indicators: punctuation, deviation and evenness indices based on bus stops, wherein the results indicate that the service reliability was relatively low. 


\section{Data and Methods}

\subsection{Description of the Study Area}

Dar es Salaam is a monocentric city; it has only one Central Business District (CBD) with the arterial roads originated from the residential area toward CBD and two ring roads. This implies that social services and economic activities are located in the city centre. The CBD is a place where government and private offices, education institutions, supermarkets, financial institutions and the port (import and export goods) are situated. Most commuters travel from the outskirts of the city towards the CBD. This situation causes rapid and unpredictable traffic flow towards the CBD during peak hours resulting in unpredictable travel time. Traffic flows in one direction in the morning hours towards the city centre and in the opposite direction in the evening exceed the capacity of the roads.

\subsection{Data Collection and Analysis}

The travel time data was collected in five main routes including Mbagala-Kariakoo (via Kilwa road), Pugu-Kariakoo (via Nyerere and Uhuru roads), Mbezi-Kariakoo (via Morogoro, Mandela, and Uhuru roads), Tegeta-Kariakoo (via Bagamoyo, Ali Hassan Mwinyi, and United Nation Road) and Kawe-Kariakoo (via Old Bagamoyo and Kawawa Roads). These routes were selected because the use of majority of commuters when travelling to the CBD and because they have a direct connection to the residential areas. Also, the routes connect the Dar es Salaam port with other parts of the country as well as routes outside the country. The travel time data was collected on working days (Mondays, Tuesdays, Wednesdays, Thursdays, and Fridays). Weekends were excluded from the sample because most people stay at home, so for that reason, traffic flow in the city becomes low. The surveys conducted between October 2018 and January 2019 managed to collect data from 28 trips per day. This means that 14 trips per day in each direction. The survey period started at 0600 to 1900 hours. The survey period was divided into two groups, off-peak hours and peak hours. For the inbound directions, peak hours started at 0600 to 1100 hours and off-peak hours started at 1100 to 1900 hours, while for the outbound directions, off-peak hours started at 06000 to 1400 hours and peak hours started at 1400 to 1900 hours. During this period, no major problems related to weather (i.e., heavy rains or floods) that might have affected travel time were reported. Travel time data was collected every one hour on five main routes in both directions (inbound and outbound) in fourteen hours, three days a week excluding weekends, this has been done in order to ensure a sufficient sample was obtained. 


\subsubsection{Sample Size}

The sample size per route obtained by the multiplication of survey time, number of directions, survey days and number of the links is in Table 1.

Table 1 Survey links and time. Source: authors

\begin{tabular}{lccccc}
\hline Corridor Name & Survey Time (hrs) & Number of Directions & Survey Days & No. of Links & Total Trips \\
\hline Tegeta-Kariakoo & 14 & 2 & 3 & 5 & 420 \\
Mbezi-Kariakoo & 14 & 2 & 3 & 3 & 252 \\
Pugu-Kariakoo & 14 & 2 & 3 & 3 & 252 \\
Mbagala-Kariakoo & 14 & 2 & 3 & 2 & 168 \\
Kawe-Kariakoo & 14 & 2 & 3 & 3 & 252 \\
\hline Total Number of trips & & & & 1344 \\
\hline
\end{tabular}

The survey time started at 06:00 to 19:00 hours for all five main routes, the data collected was travel time on each route, delay time at the intersections and waiting time of the bus at the bus stops as indicated in Table 2. The commuter buses (Daladala) operating on these routes were used as road sensors for traffic data collection.

Table 2 The main feature in the five corridors. Source: authors

\begin{tabular}{llllc}
\hline Corridor Name & \multicolumn{1}{c}{ Link Name } & Link Length (Km) & No. of Bus Stops & $\begin{array}{c}\text { No. } \\
\text { Intersections }\end{array}$ \\
\hline Tegeta-Kariakoo & Tegeta-Africana & 5.5 & 8 & 2 \\
& Africana-Mwenge & 7.2 & 9 & 4 \\
& Mwenge-Victoria & 3.4 & 6 & 2 \\
& Victoria-Mbyuni & 2.4 & 2 & 2 \\
& Mbuyuni-Kariakoo & 6.7 & 7 & 5 \\
Mbezi-Kariakoo & Mbezi-Ubungo & 12.5 & 12 & 2 \\
& Ubungo-Buguruni & 7.5 & 11 & 3 \\
Pugu-Kariakoo & Buguruni-Kariakoo & 3.6 & 7 & 2 \\
& Pugu-Aiport & 11.3 & 15 & 1 \\
& Aiport-Buguruni & 7.5 & 5 & 4 \\
Mbagala-Kariakoo & 3.6 & 8 & 2 \\
Kawe-Kariakoo & Mbagala-uhasibu & 7.0 & 10 & 1 \\
& Uhasibu-Kariakoo & 5.0 & 10 & 1 \\
& Kawe-Morocco & 7.2 & 6 & 1 \\
& Morocco-Magomeni & 3.8 & 4 & 4 \\
\hline
\end{tabular}

\subsection{Analysis of Travel Time Reliability}

Different techniques were used to analyse travel time reliability such as standard deviation and coefficient of variation. Nevertheless, standard Deviation is a handy indicator in situations where there is a need identify variability in travel times around an average value. The larger values of standard deviation, the more spread of travel time variation which results in less reliable outcomes [15]. The standard deviation calculation is indicated in the Equation 1 below:

$$
S T D=\sqrt{\frac{1}{n-1}} \sum_{i=1}^{n}(T T-M)^{2}, \quad[-]
$$


where: $S T D$ is the standard deviation [-]; $\mathrm{n}$ is the number of the trips observed; $T T$ is a travel time observation [s] and $\mathrm{M}$ is the mean travel time [s].

The Coefficient of Variation is a ratio of standard deviation over the mean travel time; it represents the percentage of the travel time variation based on mean travel time as indicated in Equation 2.

$$
C V=\frac{S T D}{M}, \quad[-]
$$

where: $C V$ is the coefficient of variation [-]; STD is the standard deviation [-] and $M$ is the mean travel time [s].

\subsubsection{The Percentile Value}

The $90^{\text {th }}$ or $95^{\text {th }}$ percentile of travel time reliability index indicate bad delay travellers experienced on the route caused by traffic congestion, weather condition, traffic control and road accidents. The 90th or 95th percentile travel times are reported in minutes to be easily understood by commuters on their daily trips

\subsubsection{Buffer Time}

Buffer time (BT) is extra time travellers add in the in-vehicle time, waiting time at bus stops and intersections. It indicates extra time to be added on the average trip based on variations, a traveller may consider these to have a high probability of arriving on time as in Equation 3. The larger the buffer time value, the higher travel time variation and the less or more reliable travel times. In general, more significant skew indicates a higher probability of extreme travel times (to the median). The large values of variation suggest that the extent of the travel time distribution is mostly related to its median value.

$$
B t=T T_{95}-T T_{m},[\mathrm{~s}]
$$

\subsubsection{Buffer Time Index (BTI)}

The Buffer Index is the ratio or percentage that passengers should add to their average travel time when planning their trips in relation to the average travel time [9]. In practice, the buffer time rate varies across the users' experience with in-vehicle travel time variation and individual requirements for arriving at the destination on time. For example, buffer index of $40 \%$ means that a traveller should count with 36 minutes as additional time for a 90 minutes average travel time to have a 95percent confidence level [4]. Buffer time index is the difference between the $95^{\text {th }}$ percentile travel time and the average travel time divided by the average travel time as in Equation 4. 


$$
B I=\frac{t t_{95}-t t_{50}}{t t_{50}} * 100,[\%]
$$

\subsubsection{Planning Time Index (PTI)}

Planning time (PT) is the total travel time added to normal travel time to be confident of arriving at the destination on time. It differs from the Buffer time index including typical delay as well as unexpected delay in the in-vehicle travel time for instance, a planning time index of 1.3 implies that passengers plan for additional $30 \%$ of travel time above the 90 or $95 \%$ travel time distribution to ensure on-time arrival. It gives the total time needed to plan to ensure $95 \%$ on-time arrival compared to normal travel time. The planning time index $(P T I)$ is calculated as the $95^{\text {th }}$ percentile travel time $\left(T T_{95}\right)$ divided by free-flow travel time $\left(T T_{\text {free-flow }}\right)$ (Equation 5)

$$
P T I=\frac{t t_{95}}{t t_{\text {Free }- \text { flow }}},[-]
$$

The 95-percentile value of travel time distribution is considered as a reference value of the BI and PTI indicators because they take into account more explicitly the extreme travel time delays compared with standard deviation. Most of the studies conducted indicate that travel time reliability can be measured using descriptive statistical data such as Buffer time [9,14,16]. For this study, travel time reliability was assessed by considering route travel time of five main routes which include in-vehicle travel time, waiting at the bus stops and waiting time at the intersections by applying standard deviation, coefficient of variations, 95th percentile travel times buffer and planning.

\section{Results and Discussion}

There are several methods to analyse travel time reliability. This study applied standard deviation, coefficient of variation, buffer time and planning time to analyse travel time reliability. This section presents the analysis of travel time reliability based on Dar es Salaams' bus operation using waiting time at the intersections and waiting at the bus stops. It applied standard deviation, coefficient of variation, buffer time and planning time to calculate travel time reliability by using data collected on the selected five main bus routes.

\subsection{Day-to-day Travel Time Variability}

Table 3 presents the Average Delay time, Standard Deviation at 95 percentile time and the coefficient of variation of all surveyed routes. The mean travel time ranges between 33.87 and 90.30 minutes on the inbound direction, and between 36.96 and 130.45 minutes on the outbound direction. The standard deviation ranges between 5.24 and 15.02 minutes on the inbound direction and between 7.89 and 23.22 minutes on the outbound direction. The coefficient of variation (CV) 
ranges between 11 and $21 \%$ for the inbound direction and between 13 and $27 \%$ for the outbound direction. Higher travel time variations observed during off-peak hours primarily in the outbound direction rather than in the inbound directions imply that the off-peak travel time especially in the outbound direction is less reliable than the inbound direction. This indicates that travel time is unreliable during the off-peak hours especially in the inbound direction which results in a low quality of transport services.

Table 3 Average travel time and standard deviation at route levels. Source: authors

\begin{tabular}{llll}
\hline Main Corridor & Parameters & Inbound-Direction & Outbound-Direction \\
\hline Mbagala -Kariakoo & Mean (min) & 33.87 & 36.98 \\
& STD (Min) & 5.24 & 7.89 \\
& 95th percentile (Min) & 35.20 & 39.42 \\
Cugu -Kariakoo & Mean (min) & 0.15 & 0.21 \\
& STD (Min) & 80.15 & 72.43 \\
& 95th percentile (Min) & 10.95 & 9.38 \\
CV & 97.20 & 85.66 \\
Mbezi-Kariakoo & Mean (min) & 0.14 & 0.13 \\
& STD (Min) & 79.05 & 81.03 \\
& 95th percentile (Min) & 8.44 & 11.53 \\
Kawe -Kariakoo & CV & 92.90 & 104.44 \\
& Mean (min) & 6.11 & 0.14 \\
& STD (Min) & 13.25 & 67.32 \\
Tegeta -Kariakoo & 95th percentile (Min) & 90.56 & 9.69 \\
& CV & 0.21 & 82.24 \\
& Mean (min) & 90.30 & 0.14 \\
& STD (Min) & 15.02 & 87.36 \\
& 95th percentile (Min) & 118.87 & 23.22 \\
& CV & 0.17 & 130.45 \\
\hline
\end{tabular}

\subsection{In-Vehicle Buffer Time}

In-vehicle Buffer time of all five surveyed routes analysed at $95 \%$ significance (Figure 2). For the inbound (Inb) and outbound (Outb) directions, the in-vehicle buffer time varies between 9.58 and 28.56 minutes and 13.23 - 43.09 minutes respectively. During the inbound off-peak (Inb-off) and outbound off-peak (Out-off) time, the buffer time varies between 9.78 and 31.06 minutes and 16.49 - 23.11 minutes respectively. Likewise, during the inbound peak hours (Inb-peak) and the outbound peak hours (Out-peak), the in-vehicle buffer time ranges from 5.89 to 53.84 minutes and from 8.06 to 38.29 minutes respectively. Higher buffer time value was observed on the Tegeta-Kariakoo route during the inbound peak hours and in outbound direction particularly in-vehicle time while the low value of buffer time was observed on the Mbagala-Kariakoo during inbound peak hours. It has been found that the travel time reliability in the peak hour is greatly affected by the route length. For example, travellers who used the Mbagala-Kariakoo route spend on average 33.87 - 36.98 minutes in the inbound and outbound directions. However, due to the travel time variation they must count with $11.2-18.87$ minutes of extra time to overcome travel time variability. 


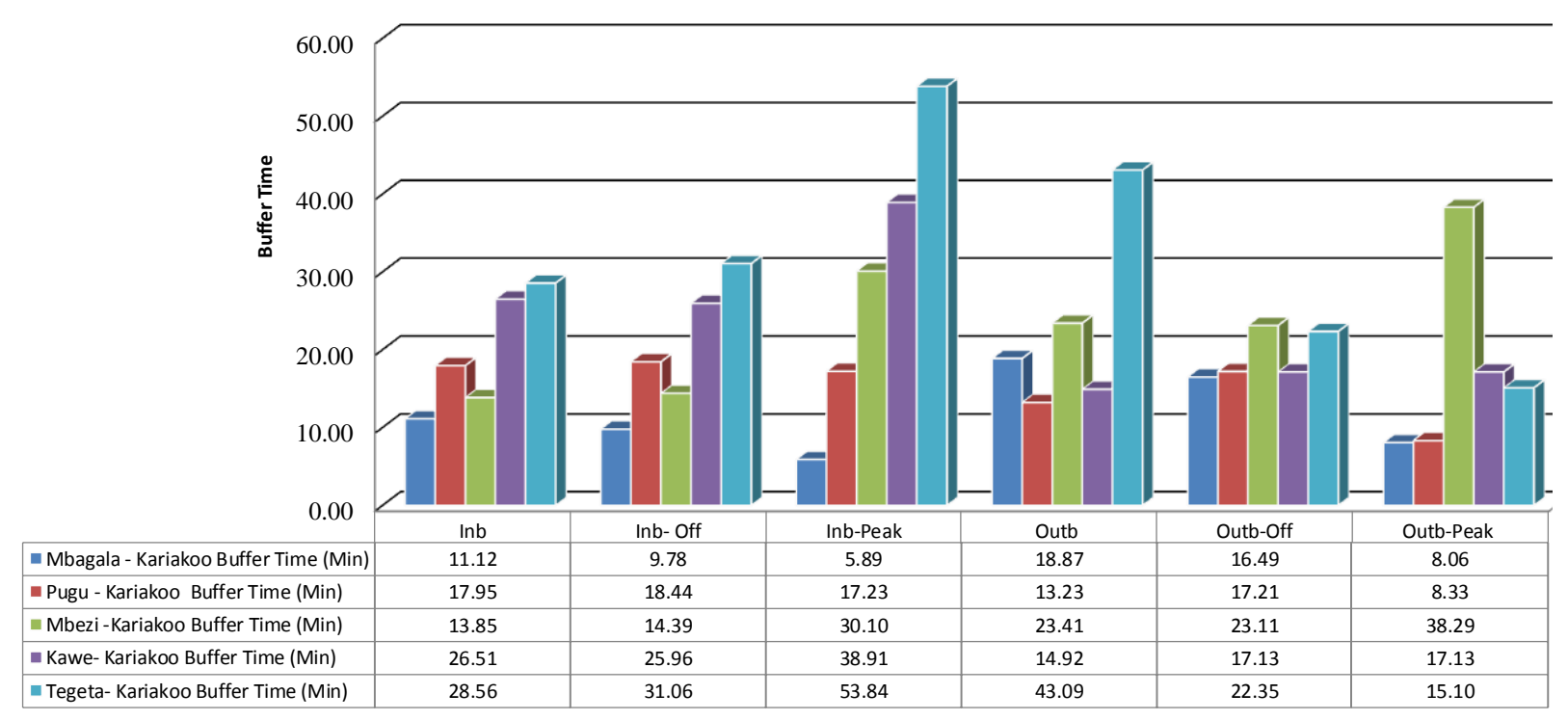

Fig. 1 In-vehicle buffer time in Dar es Salaam city. Source: authors

\subsection{In-Vehicle Planning Time}

Higher planning time values were observed in the Mbagala-Kariakoo during outbound off-peak, Pugu-Kariakoo during inbound peak hours, Mbezi-Kariakoo during outbound peak hours, KaweKariakoo during inbound off-peak and Tegeta-Kariakoo during inbound peak hours (Figure 3).

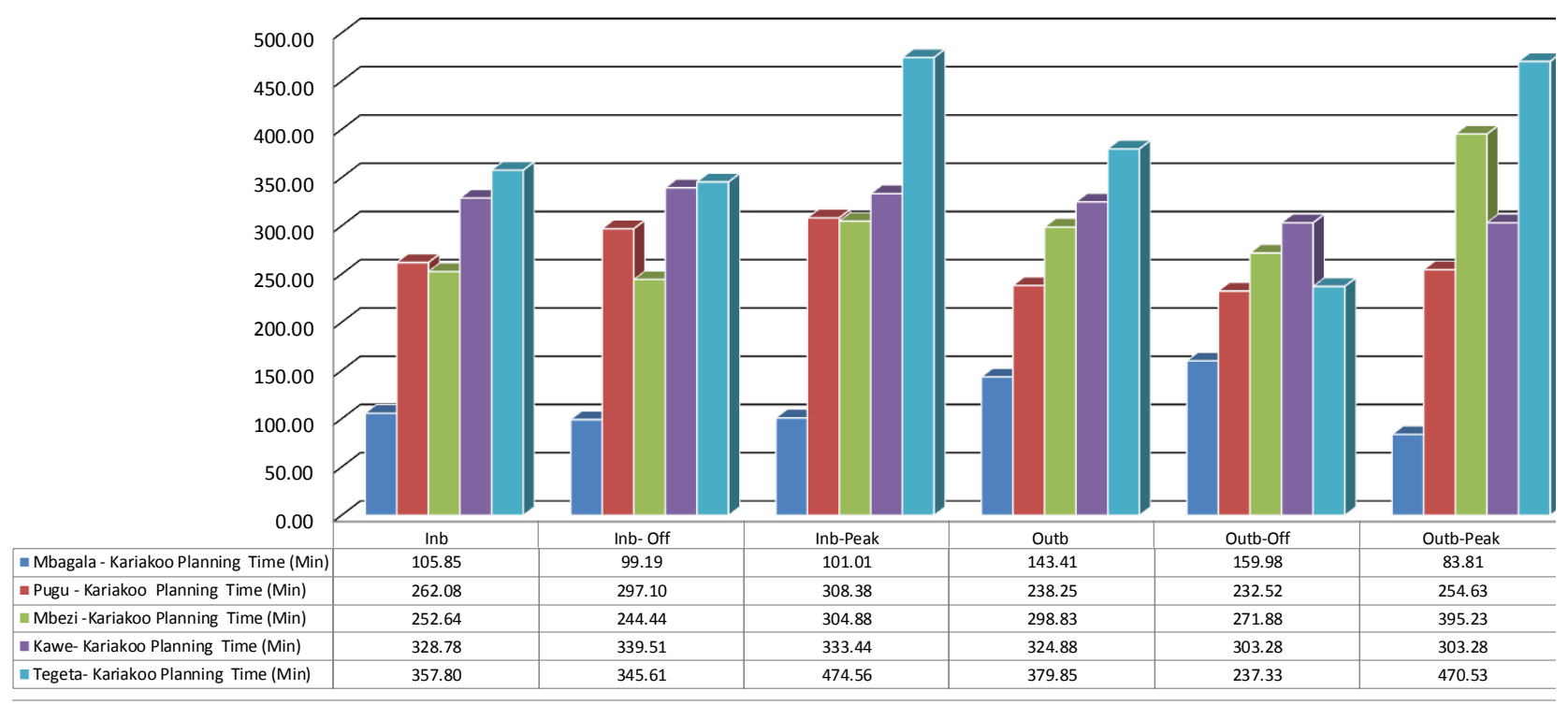

Fig. 2 In-vehicle planning time in Dar Es Salaam city. Source: authors

Also, the minimum planning time was observed on the Mbagala-Kariakoo route during outbound peak hours, Pugu-Kariakoo during outbound off-peak, Mbezi-Kariakoo during inbound off-peak, Kawe-Kariakoo during outbound off-peak hours and Tegeta-Kariakoo during outbound off-peak hours. Dar es Salaam commuters who wanted to travel from Tegeta to Kariakoo should count with 357.80 and 379.85 minutes for inbound and outbound, 345.61 and 2237.33 minutes for off-peak inbound and outbound, 474.56 and 470.53 minutes for peak hour inbound and outbound to able to arrive on time. Low value of planning time was observed in the inbound direction compared 
to the outbound direction in all corridors, the reason is that most of bus drivers drive at low speed and spend long time at the bus stops waiting for passengers.

\subsection{Route Level based on Buffer Time Index and Planning Time Index}

The In-vehicle Buffer Time Index (BTI) varies from 0.30 to 6.40 and the Planning Time Index (PTI) from 0.14 to 0.80. Higher and lower PTI values were observed in the case of Pugu-Kariakoo and Tegeta-Kariakoo while high and low BTI values were observed in the case of Pugu-Kariakoo and Mbezi-Kariakoo The results indicate that Tegeta-Kariakoo route shows low PTI value of particularly the In-vehicle time compared to the other routes while Kawe-Kariakoo shows a high value of PTI in the in-vehicle compared to the other routes. This implies that the Kawe-Kariakoo route was more congested than other routes because of more private vehicles compared to public transport vehicles (Figure 4).

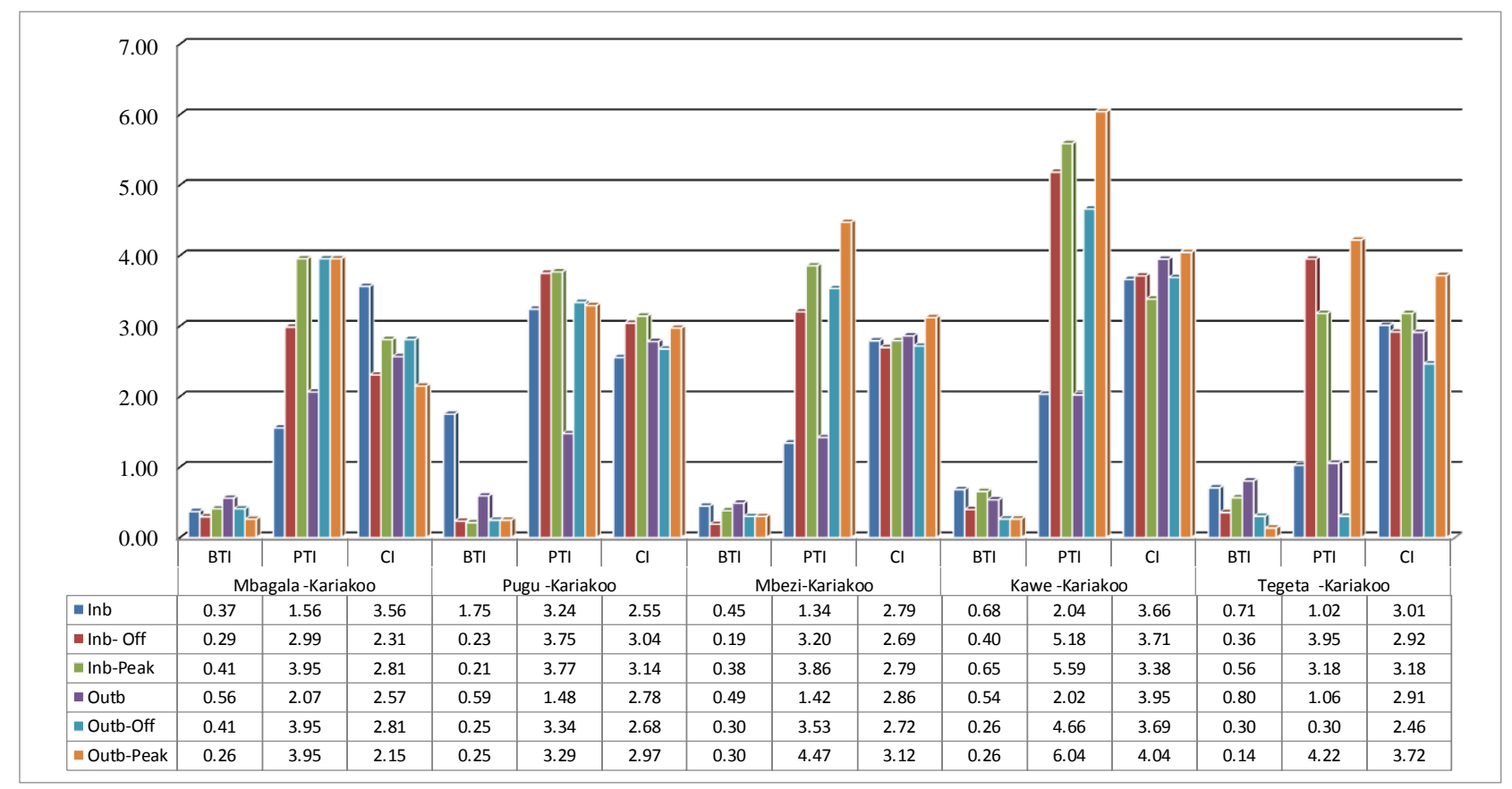

Fig. 3 Buffer time and planning time index. Source: authors

\subsection{Waiting Time Reliability at Bus Stops and Intersections}

During the inbound peak hours (Inb-Peak) and outbound peak hours the waiting time at the bus stops deviates from the mean waiting time by 1.62 - 5.02 minutes and by 1.29 - 3.26 minutes respectively. As for the inbound off-peak (Inb-off) and outbound off-peak, waiting time at the intersections deviates from the mean waiting time by $1.19-4.16$ and by $1.97-3.90$ minutes respectively (Figure 5). 


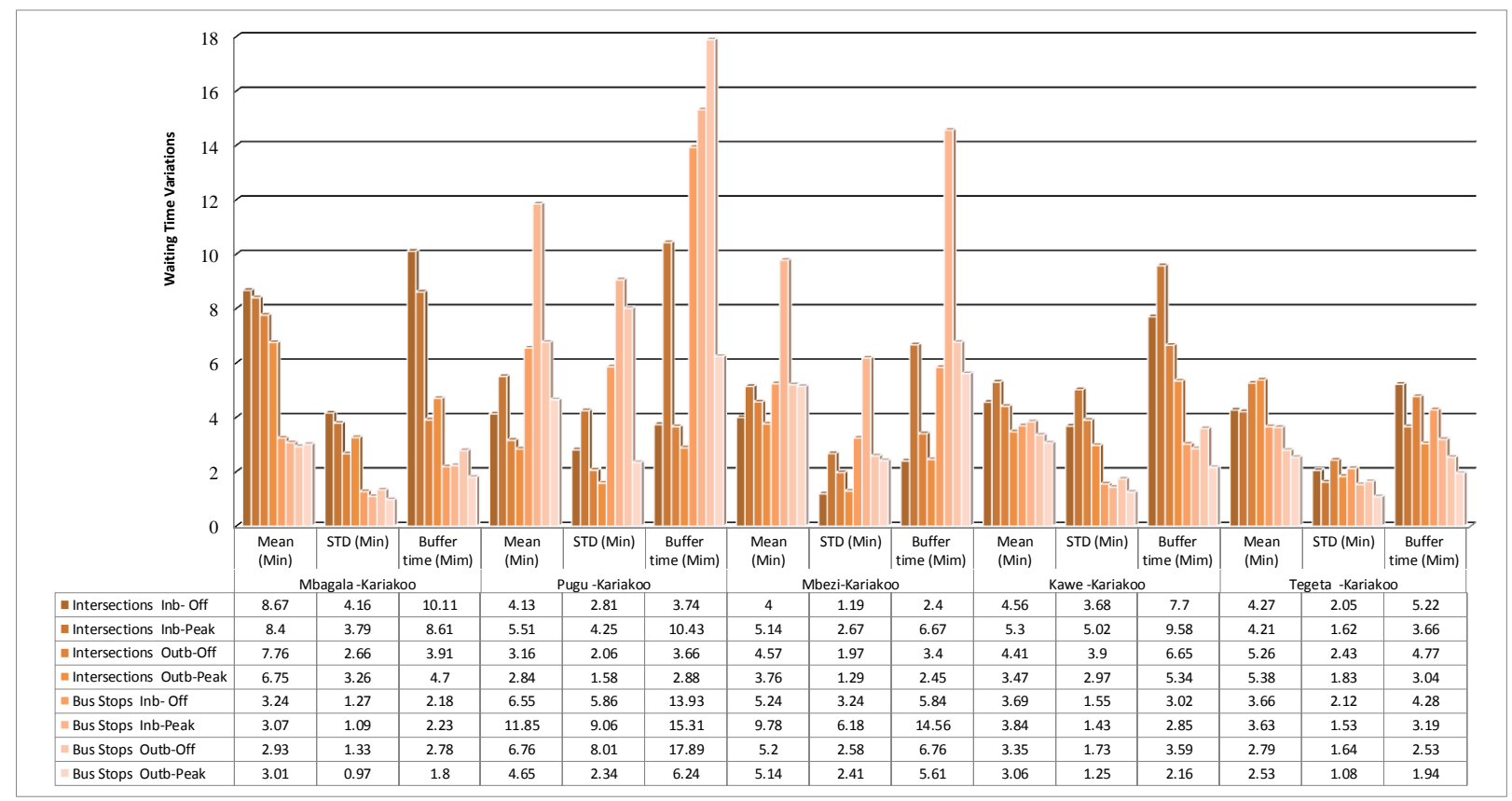

Fig. 5 Buffer time at the bus stops and intersections. Source: authors

Also, to pass through the intersection at $95 \%$ significance the passengers should count with 2.40 - 10.11 minutes of extra time during the inbound off-peak and 3.40 - 6.65 minutes during the outbound off-peak. However, during the inbound and outbound peak hours travellers should count with $3.66-10.43$ and $2.45-5.34$ minutes respectively.

\subsection{Waiting Time at Bus Stops Based on Standard Deviations and Buffer Time}

During the inbound off-peak (Inb-off) and outbound off-peak the waiting time at the bus stops deviates from the mean waiting time by 1.27 - 5.86 minutes and by 1.33 - 8.01 minutes respectively. During peak hours the waiting time differed from the mean waiting time by 1.09 9.06 minutes and $0.97-2.41$ minutes in the inbound and the outbound directions respectively. Moreover, the additional time to the mean waiting time during off-peak hours ranges from 2.18 and 13.93 minutes and between 2.53 and 17.87 minutes in the inbound and the outbound directions respectively.

A high standard deviation value was observed on the Kawe-Kariakoo route during the morning peak hours and low standard deviation value was observed on the Tegeta-Kariakoo route during the morning peak hours at the bus stops and intersections. A high value of buffer time was observed on the Pugu-Kariako route followed by the Mbezi-Kariakoo route which is probably caused by the construction work took place on these routes. However, low buffer time was observed on TegetaKariakoo route compared with the other routes despite being the longest route in the city. Also, the route has a stable passenger flow at the bus stops. These results reveal that the waiting time at the bus stops along the Tegeta-Kariakoo route is more reliable than on the other routes. 


\section{Conclusions and Recommendations}

Transport operators and engineers are aware of the importance of collecting and analysing traffic flow data for enhancing decision-making and the quality of transport services. Travellers, transport planners and policy-makers apply travel time reliability for planning, evaluating and managing transport systems. The study of travel time reliability aims to enhance policy-makers, transport planners and engineers, and operators to come up with an applicable solution for monitoring urban traffic flow. This paper analysed travel time-reliability using buffer time, standard deviation, coefficient of variation and planning time.

The study revealed that higher travel time variations experienced during off-peak hour than the peak hour. Also, high in-vehicle buffer time was observed on Tegeta-Kariakoo during the peak hour in the inbound and outbound direction while low value of in-vehicle buffer time was observed on the Mbagala-Kariakoo during peak hours in inbound. Furthermore, the low value of planning time is observed in the inbound direction especially in-vehicle time compared to the outbound direction in the city. The higher and lower value of in-vehicle PTI was observed on the Kawe-Kariakoo and Tegeta-Kariakoo routes respectively compared to the other routes. Low buffer time was observed at the bus stops on the Tegeta-Kariakoo route compared to the other routes despite being the longest route in the city.

The waiting time at the bus stops and intersections along the Tegeta-Kariakoo route is more reliable compared to other routes. There are several indicators used to analyse travel time reliability; however, this study considered only five indicators to evaluate travel time reliability in Dar es Salaam. Therefore, more indicators can be applied to evaluate travel time reliability in to obtain more reliable traffic flow information. Furthermore, the data used to analyse travel time reliability was collected in three days only, which may not be sufficient. However, the findings of this study provide potential useful information for road users, bus operators and urban transport planners. Therefore, further studies should consider collecting more data and including more variables such as weather conditions, distance, traffic flow and road incidents in order to improve travel time reliability analyses.

\section{Acknowledgement}

The authors would like to acknowledge the support given by the National Institute of Transport and JICA for issuing permission to utilize their data for this study.

\section{References}

[1] Kieu, L., Bhaskar A. \& Chung, E. (2013). Empirical evaluation of public transport travel time variability. Australasian Transport Research Forum Proceedings, 2 - 4 October 2013, 
Brisbane, Australia.

[2] Tirachini, A., Hensher A., D. \& M., Bliemer J., C. (2014). Accounting for travel time variability in the optimal pricing of cars and buses. Transportation. DOI: $10.1007 / \mathrm{s} 11116$ 014-9515-8.

[3] Zhenliang, Z., Ferrira, M.L \& Mesbah. M. (2015). Modeling distributions of travel time variability for bus operations, Journal of Advance Transport44, 267-283.

[4] OECD. (2010). Improving reliability on surface transport networks. Journal Transport Center 9(2), 1-165.

[5] Zhu, X., Fan Y., Zhang F., \& Ye X. (2018). Multiple-Factor Based Sparse Urban Travel Time Prediction. Appl. Sci. 8, 1-18. DOI:10.3390/app8020279.

[6] Anil, K.B., Lelitha V. \& S.S.C. (2017). A hybrid model based method for bus travel time estimation. Journal of Intelligent Transportation Systems22(5), 390-406. DOI: 10.1080/15472450.2017.1378102.

[7] Torrisi, V., Ignaccolo M. \& Inturri G. (2017). Estimating travel time reliability in urban areas through a dynamic simulation model. Transport Research Procedia27, 857-864.

[8] Vanderval, R., Annta L., Guerre H. \& Margiotta J. (2014). Incorporating Reliability Performance Measures into the Transportation Planning and Programming Processes. Washington, DC: The National Academies Press. DOI: 10.17226/22594.

[9] Bhouri, N., Aron M.\& Scemama G. (2016). Travel time reliability with and without the dynamic use of hard shoulder: Field assessment from a French motorway. Journal of Traffic Transport Engineering3(6), 520-530.

[10] Charlotte, C., Helene L. M. \& Sandra B. (2017). Empirical estimation of the variability of travel time. Transport Research Procedia25, 2769-2783.

[11] Durán-Hormazábal, E. \& Tirachini A. (2016). Estimation of travel time variability for cars, buses, metro and door-to-door public transport trips in Santiago. Transport Economic 59, 2639.

[12] Mehran, B. (2009). Considering Travel Time Reliability and Safety for Evaluation of Congestion Relief. IATSS Research1(33),55-70.

[13] Taylor, M.A.P. (2012). Modelling Travel Time Reliability with the Burr Distribution. Procedia Social Behaviour. Science54, 75-83.

[14] Chen, X., Yu L., Zhang Y. \& Guo J. (2009). Analyzing urban bus service reliability at the stop, route, and network levels. Transport Research Part A43(8), 722-734.

[15] Guessous, Y., Aron M., Bhouri N. \& Cohen, S. (2014). Estimating Travel Time Distribution Under Different Traffic Conditions. Transport. Research Procedia 3, 339-348.

[16] FDOT, (2016). Guide to Planning for Travel Time Reliability, Florida Department of Transportation 605 Suwannee Street. 